\title{
Beyond the rainbow: effects from pion back-coupling
}

\author{
Christian S. Fischer ${ }^{1,2}$ and Richard Williams ${ }^{1}$ \\ ${ }^{1}$ Institute for Nuclear Physics, Darmstadt University of Technology, \\ Schlossgartenstraße 9, 64289 Darmstadt, Germany \\ ${ }^{2}$ Gesellschaft für Schwerionenforschung mbH, Planckstr. 1 D-64291 Darmstadt, Germany.
}

(Dated: October 26, 2018)

\begin{abstract}
We investigate hadronic unquenching effects in light quarks and mesons. To this end we take into account the back-coupling of the pion onto the quark propagator within the non-perturbative continuum framework of Schwinger-Dyson equations (SDE) and Bethe-Salpeter equations (BSE). We improve on a previous approach by explicitly solving both the coupled system of DSEs and BSEs in the complex plane and the normalisation problem for Bethe-Salpeter kernels depending on the total momentum of the meson. As a result of our study we find considerable unquenching effects in the spectrum of light pseudoscalar, vector and axial-vector mesons.
\end{abstract}

PACS numbers: 12.38.Aw, 12.38.Gc, 12.38.Lg, 14.65.Bt

Keywords: Dynamical chiral symmetry breaking, light mesons, pion cloud

\section{INTRODUCTION}

In hard scattering events mesons and baryons can be viewed as bound states built up from partonic constituents, i.e. quarks and gluons. This picture changes at low energies, where hadronic effects play a more prominent rôle in the nonperturbative structure of hadrons. Of particular importance are pion cloud effects which e.g. have a direct impact on the spin structure of the proton [1]. Thus they need to be incorporated in bound-state calculations aiming at a realistic description of mesons and baryons.

For the spectrum of light pseudoscalar mesons, it is the axial-vector Ward-Takahashi identity that governs the pattern of dynamical symmetry breaking, rather than the intricacies of confinement or infrared specifics of QCD. Within the framework of Schwinger-Dyson (SDE) and Bethe-Salpeter (BSE) equations, this realisation has led to much phenomenological success in describing pseudoscalar and vector mesons [2, 3, 4], with boundstate masses, decay constants and electromagnetic formfactors determined [5, 6] with surprising agreement to experiment 7].

The success of this description partly comes from the vector-vector coupling of the rainbow-ladder truncation, where the resultant spin-coupling to these $S$-wave mesons is dominant. For $P$-wave states however, such as the scalar and axial-vector mesons, a richer tensor structure is required from the quark-gluon vertex to properly account for the observed spectrum. To this end, the scalar part of the quark-gluon vertex, whose importance regarding confinement has been highlighted in [8], may be the most relevant for introducing a spin-dependence to the interaction. We expect any beyond-the-rainbow study that generates such terms to have an effect on the spectrum of light mesons.

However, there arise further complications in describing the scalar and axial-vector sector. Not only are models employing the rainbow-ladder truncation too attractive, yielding masses of $800-900 \mathrm{MeV}$ [4, 9], but are these mesons even $q \bar{q}$ bound states? For the scalars we have tetraquarks, multi-meson molecules, glueballs and the inevitable hybrids as candidates (see e.g. [10,11]). Indeed, these exotics are not restricted to the scalar sector, for the $a_{1}$ may be well described as a coupled-channel meson molecule 12, amongst other equally plausible descriptions [13, 14]. Clearly there is much work to be done in providing a dynamical partonic description of these 'mesons', not least in understanding the fundamental interactions between quarks and gluons.

As a further step in this direction we concentrate in this work on improving our model description of $\bar{q} q$ boundstates. Based on an approximation scheme for the quarkgluon interaction developed in [15] and [16] we study the pion back-reaction on the quarks and light mesons in the covariant Schwinger-Dyson/Bethe-Salpeter approach to Landau gauge QCD [17, 18]. As a consequence we take into account pion cloud effects in the light meson spectrum. To this end we solve the coupled system of quark Schwinger-Dyson equations and BetheSalpeter equations for pseudoscalar, vector and axialvector mesons in the complex plane. This provides for an important technical progress compared to the calculations in Ref. [15], where the so-called real-value approximation has been used. As a further technical complication we then have to solve the normalisation problem for Bethe-Salpeter amplitudes generated by a kernel depending on the total momentum of the meson.

The paper is organized as follows. In Sec. III we summarise our approximation scheme for the quark-gluon interaction developed in detail in [15, 16]. We specify our model interaction and discuss the technical complications that appear in the normalisation condition for the boundstate amplitudes. We explicitly verify that our interaction satisfies the axial-vector Ward-Takahashi identity. In Sec. III] we then provide the details of our calculation and present our results. We conclude with an outlook in Sec. IV. Some technical discussions are relegated to an appendix. 


\section{THE QUARK-GLUON INTERACTION}

\section{A. General truncation}

In the past, a widely used practical approximation scheme for the coupled system of quark-SDE and mesonBSE is the rainbow-ladder approximation. Its most important property is that it satisfies the non-singlet axialvector Ward-Takahashi identity (axWTI) which guarantees that the pion in the chiral limit is both a Goldstone boson and a bound state of massive constituents. This scheme has a history of remarkable successes (summarised e.g. in [17]). However, there are also shortcomings that limit the credibility of such an approximation. Consequently, several efforts have been made to extend this scheme, see e.g. [19, 20, 21, 22, 23, 24]. In the following, we will continue the strategy employed in [15] to extend the rainbow-ladder scheme by taking into account pion cloud effects in the quark-gluon interaction. These effects can be accounted for by a particular class of interaction diagrams that only appear in the unquenched theory. Our strategy is complementary to the one followed in 25], where a different class of unquenching effects have been investigated. The aim there was to provide for hadronic intermediate states in bound-state calculations which generate a finite width of meson spectral functions. It is also complementary to the investigations reported in [19, 26], where unquenching effects in the gluon polarization have been considered.

We start with the SDE for the quark propagator, given diagrammatically in Fig. 1. With the fully dressed in-

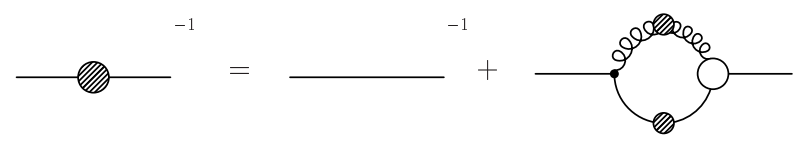

FIG. 1: The Schwinger-Dyson equation for the fully dressed quark propagator

verse quark propagator $S^{-1}(p)=i \not p A\left(p^{2}\right)+B\left(p^{2}\right)$ and its bare counterpart $S_{0}^{-1}(p)=i \not p+m$, the equation is given by

$$
\begin{aligned}
S^{-1}(p) & =Z_{2} S_{0}^{-1}(p) \\
+ & g^{2} C_{F} Z_{1 F} \int \frac{d^{4} q}{(2 \pi)^{4}} \gamma_{\mu} S(q) \Gamma_{\nu}(q, k) D_{\mu \nu}(k),
\end{aligned}
$$

with $k=p-q$, the Casimir $C_{F}=\left(N_{c}^{2}-1\right) /\left(2 N_{c}\right)$ and the renormalization factors $Z_{1 F}=Z_{2} / \widetilde{Z}_{3}$ of the quark-gluon vertex, $Z_{2}$ of the quark propagator and $\widetilde{Z}_{3}$ of the ghost dressing function. The quark dressing functions $A\left(p^{2}\right)$ and $B\left(p^{2}\right)$ can be recombined into the quark mass $M\left(p^{2}\right)=B\left(p^{2}\right) / A\left(p^{2}\right)$ and the quark wave function $Z_{f}\left(p^{2}\right)=1 / A\left(p^{2}\right)$. The quark self-energy depends on the fully dressed quark-gluon vertex $\Gamma_{\nu}(q, k)$ and the gluon

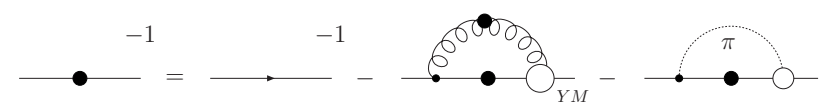

FIG. 2: The approximated Schwinger-Dyson equation for the quark propagator with effective one-gluon exchange and onepion exchange.

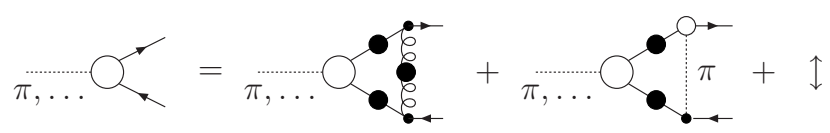

FIG. 3: The Bethe-Salpeter equation corresponding to the quark self-energy of Fig. 2 The up-down arrow indicates an averaging procedure of the pion-exchange diagram with its counterpart where the upper pion-quark vertex is bare and the lower one dressed.

propagator

$$
D_{\mu \nu}(k)=\left(\delta_{\mu \nu}-\frac{k_{\mu} k_{\nu}}{k^{2}}\right) \frac{Z\left(k^{2}\right)}{k^{2}},
$$

with the gluon dressing function $Z\left(k^{2}\right)$. Throughout this paper we will work in the Landau gauge, which guarantees the transversality of (2). The explicit expression for $Z\left(k^{2}\right)$ used in this work will be given in Sec. IB,

The key quantity in the SDE for the quark propagator is the quark-gluon vertex. It satisfies its own Schwinger-Dyson equation which contains various basically unknown four-point functions. The structure of the Yang-Mills part of this equation and the resulting pattern of dynamical chiral symmetry breaking has been discussed recently in detail in Ref. [8]. Here we are interested in the hadronic contributions to this vertex. These have been discussed in Ref. [15] and [16], where two slightly different approximations in terms of one-pion exchange have been constructed. Whereas the scheme of [15] resulted in a very strong back-reaction of the pion onto the quark propagator in disagreement with corresponding lattice results, the modified scheme in [16] seems to represent these effects quantitatively correctly. We therefore resort to the latter scheme here, whose diagrammatic expressions for the quark-SDE and the corresponding Bethe-Salpeter equation for quark-antiquark bound states are given in Figs. 2 and 3 ,

The effective self-interaction of the quark is thus given by a pure Yang-Mills part and a hadronic part approximated by one-pion exchange. The dressed coupling of the pion to the quark line is hereby described by its BetheSalpeter vertex function, whereas the bare one is given by $\tau^{j} \gamma_{5} Z_{2}$ with the generators $\tau^{j}$ of flavour $\mathrm{SU}(2)$.

Certainly it is important that the explicit form of both the Yang-Mills part and the pionic part of the interaction is such that the axial-vector Ward-Takahashi identity is satisfied. Naturally, as a first guess for the Yang-Mills 
part one takes a rainbow-ladder form of the interaction, i.e. one chooses a model for the fully dressed Yang-Mills part of the quark-gluon vertex $\Gamma_{\nu}^{\mathrm{YM}}$ of the form

$$
\Gamma_{\nu}^{Y M}\left(p_{1}, p_{2}, p_{3}\right)=\gamma_{\nu} Z_{2} / \widetilde{Z}_{3} \Gamma^{Y M}\left(p_{3}^{2}\right)
$$

where we denote the quark momenta by $p_{1}$ and $p_{2}$ and the gluon momentum by $p_{3}$. The explicit expression for $\Gamma^{\mathrm{YM}}\left(p_{3}^{2}\right)$ is detailed below. A similar form has been used in [27, 28], where quenched lattice results for the quark propagator have been reproduced. Note, however, that (3) involves only the $\gamma_{\nu}$-part of the tensor structure of the full Yang-Mills part of the vertex. It has been shown in the analysis of the full quark-gluon vertex of Ref. [8] that such a model cannot capture all essentials of dynamical chiral symmetry breaking. Indeed, from the results of this work we will draw the very same conclusion from our calculation of the light meson spectrum.

In general one can decompose the quark-gluon vertex $\Gamma^{\nu}\left(p_{1}, p_{2}, p_{3}\right)$ into 12 different tensor structures, given by

$$
\Gamma_{\nu}\left(p_{1}, p_{2}, p_{3}\right)=\sum_{i=1}^{12} \lambda_{i}\left(p_{1}, p_{2}, p_{3}\right) L_{\nu}^{i}\left(p_{1}, p_{2}, p_{3}\right)
$$

The details of this basis are given in Ref. [29]. Here, we only note that $L_{\nu}^{1}=\gamma_{\nu}$; the explicit forms of the other structures are not needed. In principle, all twelve tensor structures are important in the infrared and intermediate momentum regime [8, 30]. We have verified by projection methods that the pion-exchange diagram in the vertex-SDE indeed contributes to all these structures. Therefore the resulting one-pion contribution in the propagator SDE Fig. 2 generates unquenching effects in all components of the quark-gluon interaction.

An explicit expression for the one pion-exchange kernel that satisfies the axial-vector Ward-Takahashi identity has been constructed in [15]. Here we give a similar construction which takes into account the modifications suggested in [16]. The resulting SDE for the quark propagator is given by

$$
\begin{aligned}
S^{-1}(p)=Z_{2} S_{0}^{-1}(p) & +g^{2} C_{F}\left(Z_{2}\right)^{2} \int \frac{d^{4} q}{(2 \pi)^{4}} \gamma_{\mu} S(q) \gamma_{\nu}\left(\delta_{\mu \nu}-\frac{k_{\mu} k_{\nu}}{k^{2}}\right) \frac{Z\left(k^{2}\right) \Gamma_{\mathrm{YM}}\left(k^{2}\right)}{k^{2}} \\
& -3 \int \frac{d^{4} q}{(2 \pi)^{4}}\left[Z_{2} \gamma_{5} S(q) \Gamma_{\pi}\left(\frac{p+q}{2} ; q-p\right)+Z_{2} \gamma_{5} S(q) \Gamma_{\pi}\left(\frac{p+q}{2} ; p-q\right)\right] \frac{D_{\pi}\left(k^{2}\right)}{2}
\end{aligned}
$$

with $k=p-q$ and $D_{\pi}(k)=\left(k^{2}+M_{\pi}^{2}\right)^{-1}$ being the pion propagator. The factor 3 in front of the pion contribution stems from the flavour trace and represents contributions from $\pi_{+}, \pi_{-}$and $\pi_{0}$. These are treated on equal footing in the isospin-symmetric limit adopted here.

The corresponding expression for the Bethe-Salpeter equation for light mesons reads

$$
\Gamma_{t u}^{(\mu)}(p ; P)=\int \frac{d^{4} k}{(2 \pi)^{4}}\left\{K_{t u ; r s}^{\mathrm{YM}}(p, k ; P)+K_{t u ; r s}^{\mathrm{pion}}(p, k ; P)\right\}\left[S\left(k_{+}\right) \Gamma^{(\mu)}(k ; P) S\left(k_{-}\right)\right]_{s r}
$$

with the kernels $K_{t u ; r s}^{\mathrm{YM}}$ and $K_{t u ; r s}^{\mathrm{pion}}$ given by

$$
\begin{aligned}
K_{t u ; s r}^{\mathrm{YM}}(q, p ; P) & =\frac{g^{2} Z\left(k^{2}\right) \Gamma^{\mathrm{YM}}\left(k^{2}\right) Z_{1 F}}{k^{2}}\left(\delta_{\mu \nu}-\frac{k_{\mu} k_{\nu}}{k^{2}}\right)\left[\frac{\lambda^{a}}{2} \gamma_{\mu}\right]_{t s}\left[\frac{\lambda^{a}}{2} \gamma_{\nu}\right]_{r u}, \\
K_{t u ; r s}^{\mathrm{pion}}(q, p ; P) & =\frac{1}{4}\left[\Gamma_{\pi}^{j}\right]_{r u}\left(\frac{p+q-P}{2} ; p-q\right)\left[Z 2 \tau^{j} \gamma_{5}\right]_{t s} D_{\pi}(p-q) \\
& +\frac{1}{4}\left[\Gamma_{\pi}^{j}\right]_{r u}\left(\frac{p+q-P}{2} ; q-p\right)\left[Z 2 \tau^{j} \gamma_{5}\right]_{t s} D_{\pi}(p-q) \\
& +\frac{1}{4}\left[\Gamma_{\pi}^{j}\right]_{t s}\left(\frac{p+q+P}{2} ; p-q\right)\left[Z 2 \tau^{j} \gamma_{5}\right]_{r u} D_{\pi}(p-q) \\
& +\frac{1}{4}\left[\Gamma_{\pi}^{j}\right]_{t s}\left(\frac{p+q+P}{2} ; q-p\right)\left[Z 2 \tau^{j} \gamma_{5}\right]_{r u} D_{\pi}(p-q) .
\end{aligned}
$$




\begin{tabular}{ccc|cc} 
& Component & $0^{-}$ & $0^{+}$ \\
\hline$T_{1}(p ; P)$ & $:$ & $\mathbb{1}$ & $\circ$ & $\circ$ \\
$T_{2}(p ; P)$ & $:$ & $-i \not P$ & $\circ$ & $\bullet$ \\
$T_{3}(p ; P)$ & $:$ & $-i \not p$ & $\bullet$ & $\circ$ \\
$T_{4}(p ; P)$ & $:$ & {$[\not p, \not P]$} & $\circ$ & $\circ$
\end{tabular}

TABLE I: The four Dirac structures describing a meson of spin $J=0$. For the pseudoscalar, these have an associated factor of $\gamma_{5}$ to account for parity. Circles indicate that the component is part of the basis decomposition of the meson, with filled circles indicating that the component is multiplied by $(p \cdot P)$. This ensures that for equal-mass constituents a Chebyshev decomposition of even order need only been employed for the scalar amplitudes $F_{i}$.

\begin{tabular}{llc|c|cc} 
& Component & $1^{-}$ & $1^{++}$ & $1^{+-}$ \\
\hline$T_{1}^{\mu}(p ; P):$ & $i \gamma_{T}^{\mu}$ & $\circ$ & $\circ$ & \\
$T_{2}^{\mu}(p ; P):$ & $\gamma_{T}^{\mu} \not P$ & $\circ$ & $\bullet$ & \\
$T_{3}^{\mu}(p ; P):$ & $-\gamma_{T}^{\mu} \not p+p_{T}^{\mu} \mathbb{1}$ & $\bullet$ & $\circ$ & \\
$T_{4}^{\mu}(p ; P):$ & $: \gamma_{T}^{\mu}[\not P, \not p]+2 i p_{T}^{\mu} \not \supset$ & $\circ$ & $\circ$ & \\
$T_{5}^{\mu}(p ; P):$ & $p_{T}^{\mu} \mathbb{1}$ & $\circ$ & & $\circ$ \\
$T_{6}^{\mu}(p ; P):$ & $i p_{T}^{\mu} \not P$ & $\bullet$ & & $\circ$ \\
$T_{7}^{\mu}(p ; P):$ & $-i p_{T}^{\mu} \not p$ & $\circ$ & & $\bullet$ \\
$T_{8}^{\mu}(p ; P):$ & $p_{T}^{\mu}[\not P, \not p]$ & $\circ$ & & $\circ$
\end{tabular}

TABLE II: The eight Dirac structures describing a meson of spin $J=1$. For the axial-vectors, there is an additional factor of $\gamma_{5}$ to account for parity. Circles indicate that the component is included in the basis decomposition of the meson, with filled circles indicating the component is multiplied by $(p \cdot P)$. This ensures that an even Chebyshev decomposition is sufficient for equal-mass constituents. The subscript $T$ indicates transversality with respect to the total momentum.

Here $\Gamma^{(\mu)}(p ; P)$ is the Bethe-Salpeter vertex function of a quark-antiquark bound state specified below, whereas $\Gamma_{\pi}^{j}$ with flavour index $j$ is the corresponding vertex function of a pion. The momenta $k_{+}=k+P / 2$ and $k_{-}=k-P / 2$ are such that the total momentum $P$ of the meson is given by $P=k_{+}-k_{-}$and the relative momentum $k=$ $\left(k_{+}+k_{-}\right) / 2$. The Latin indices $(t, u, r, s)$ of the kernels refer to colour, flavour and Dirac structure.

The Bethe-Salpeter vertex function $\Gamma(p ; P)^{(\mu)}$ can be decomposed into at most eight Lorentz and Dirac structures. The structure is constrained by the transformation properties under CPT of the meson we wish to describe [31]. In particular, our pseudoscalar, scalar and vector have quantum numbers $J^{P}$ of $0^{-}, 0^{+}$and $1^{-}$, respectively. The axial-vector can be parameterised in two different ways, dependent on its transformation under charge conjugation, $J^{P C}=1^{++}$and $1^{+-}$.

Taking this into consideration, we can write down a general basis for each desired meson amplitude,

$$
\Gamma_{M}^{(\mu)}(p ; P)= \begin{cases}\sum_{i} \quad F_{i}(p ; P) T_{i}^{(\mu)}(p ; P) & J^{P}=0^{+}, 1^{-} \\ \sum_{i} \gamma_{5} F_{i}(p ; P) T_{i}^{(\mu)}(p ; P) & J^{P}=0^{-}, 1^{+}\end{cases}
$$

where the components $T_{i}^{(\mu)}$ are given in Tables $\square$ and II.

In particular, the pion is given by the following form

$$
\begin{aligned}
\Gamma_{\pi}(p ; P) & =\gamma_{5}\left[F_{1}(p ; P)-i \not P F_{2}(p ; P)\right. \\
-i \not p & \left.(p \cdot P) F_{3}(p ; P)-[\not P, \not p] F_{4}(p ; P)\right] .
\end{aligned}
$$

\section{B. Model details}

Next we need to specify the details of our model interaction. For the Yang-Mills part of the interaction we choose two different model ansätze that have been employed already in previous works. Here, the gluon dressing function $Z\left(k^{2}\right)$ from (2) and the Yang-Mills part $\Gamma^{\mathrm{YM}}\left(k^{2}\right)$ of the quark-gluon vertex Eq. (3) are combined and represented by a single function. For the MarisTandy (MT) model [4] this function is given by

$$
\begin{aligned}
Z\left(k^{2}\right) \Gamma^{\mathrm{YM}}\left(k^{2}\right)= & \frac{4 \pi}{g^{2}}\left(\frac{\pi}{\omega^{6}} D q^{4} \exp \left(-q^{2} / \omega^{2}\right)\right. \\
+ & \frac{2 \pi \gamma_{m}}{\log \left(\tau+\left(1+q^{2} / \Lambda_{\mathrm{QCD}}^{2}\right)^{2}\right)} \\
& \left.\times\left[1-\exp \left(-q^{2} /\left[4 m_{t}^{2}\right]\right)\right]\right),
\end{aligned}
$$

with

$$
\begin{aligned}
& m_{t}=0.5 \mathrm{GeV} \quad, \quad \tau=\mathrm{e}^{2}-1 \\
& \gamma_{m}=12 /\left(33-2 N_{f}\right), \quad \Lambda_{\mathrm{QCD}}=0.234 \mathrm{GeV} \text {. }
\end{aligned}
$$

This model has been employed successfully in various calculations of meson spectra, form factors and decay constant within a rainbow-ladder truncation; see [17, 22] for overviews of the results. The remaining parameters $D$, $\omega$ and the quark mass are fitted to pion observables, and detailed in Sec. III.

The second model we employ for the Yang-Mills part of the quark-gluon interaction represents the infrared structure of both the gluon dressing function $Z\left(k^{2}\right)$ and the quark-gluon vertex $\Gamma^{\mathrm{YM}}\left(k^{2}\right)$, in quenched approximation (up to effects due to hairpin diagrams) as calculated in Refs. [8, 32]. It has been used to show that infrared divergences in vertex functions can account for the topological 
mass of the $\eta^{\prime}$-meson [33], and is given by

$$
\begin{gathered}
Z\left(k^{2}\right)=\left(\frac{k^{2}}{k^{2}+\Lambda_{\mathrm{QCD}}^{2}}\right)^{2 \kappa}\left(\frac{\alpha_{\mathrm{fit}}\left(k^{2}\right)}{\alpha_{\mu}}\right)^{-\gamma}, \\
\Gamma^{\mathrm{YM}}\left(k^{2}\right)=\left(\frac{k^{2}}{k^{2}+d_{2}}\right)^{-1 / 2-\kappa} \\
\times\left(\frac{d_{1}}{d_{2}+k^{2}}+\frac{k^{2} d_{3}}{d_{2}^{2}+\left(k^{2}-d_{2}\right)^{2}}+\frac{k^{2}}{\Lambda_{\mathrm{QCD}}^{2}+k^{2}}\right. \\
\left.\times\left[\frac{4 \pi}{\beta_{0} \alpha_{\mu}}\left(\frac{1}{\log \left(\frac{k^{2}}{\Lambda_{\mathrm{QCD}}^{2}}\right)}-\frac{\Lambda_{\mathrm{QCD}}^{2}}{k^{2}-\Lambda_{\mathrm{QCD}}^{2}}\right)\right]^{-2 \delta}\right)
\end{gathered}
$$

with the gluon momentum $k^{2}$, the one-loop value $\gamma=$ $\left(-13 N_{c}+4 N_{f}\right) /\left(22 N_{c}-4 N_{f}\right)$ for the anomalous dimension of the re-summed gluon propagator, the corresponding one $\delta=\frac{-9 N_{c}}{44 N_{c}-8 N_{f}}$ for the ghost dressing function and $\alpha_{\mu}=0.2$ at the renormalisation scale $\mu^{2}=170 \mathrm{GeV}^{2}$. We use $\Lambda_{\mathrm{QCD}}^{2}=0.52 \mathrm{GeV}^{2}$ similar to the scale obtained in Ref. 32]. The infrared exponent $\kappa$ has been determined analytically in 34, 35] and is given by $\kappa=$ $(93-\sqrt{1201}) / 98 \simeq 0.595$. Note that this form is slightly modified to that reported in [16, 33], allowing more freedom in separating the scales in the quark-gluon vertex and the gluon propagator. The parameter $d_{1}$ sets the strength of the interaction in the infrared. The parameters $d_{2}$ and $d_{3}$ determine the location and size of a term added to give additional integrated strength in the intermediate momentum regions. These three parameters, together with the current quark mass, are constrained by pion observables and the generation of a topological charge in the pseudoscalar isosinglet channel. The running coupling from the ghost-gluon vertex, $\alpha_{\mathrm{fit}}\left(p^{2}\right)$, is parameterised such that the numerical results for Euclidean scales are accurately reproduced [32]:

$$
\begin{aligned}
\alpha_{\mathrm{fit}}\left(p^{2}\right)= & \frac{\alpha_{s}(0)}{1+p^{2} / \Lambda_{\mathrm{QCD}}^{2}}+\frac{4 \pi}{\beta_{0}} \frac{p^{2}}{\Lambda_{\mathrm{QCD}}^{2}+p^{2}} \\
& \left(\frac{1}{\ln \left(p^{2} / \Lambda_{\mathrm{QCD}}^{2}\right)}-\frac{1}{p^{2} / \Lambda_{\mathrm{QCD}}^{2}-1}\right) .
\end{aligned}
$$

Here $\beta_{0}=\left(11 N_{c}-2 N_{f}\right) / 3$, and $\alpha_{S}(0)$ is the fixed point in the infrared, calculated to be $\alpha_{S}(0)=8.915 / N_{c}$ for our choice of $\kappa$. In this work, we will take $N_{f}=2$.

The resulting two effective interactions from the MarisTandy model (11) and our soft-divergence model (12) are plotted against each other in Fig. 4. The ultraviolet running of both interactions are the same except for differences in the scale employed. Whereas the Maris-Tandy model used a scale corresponding to the $\overline{M S}$ scheme, we are working in a momentum subtraction scheme where the scale is larger by more than a factor of 3 . Both interactions share an enhancement in the intermediate momentum region which provides the necessary interaction strength for dynamical chiral symmetry breaking. The

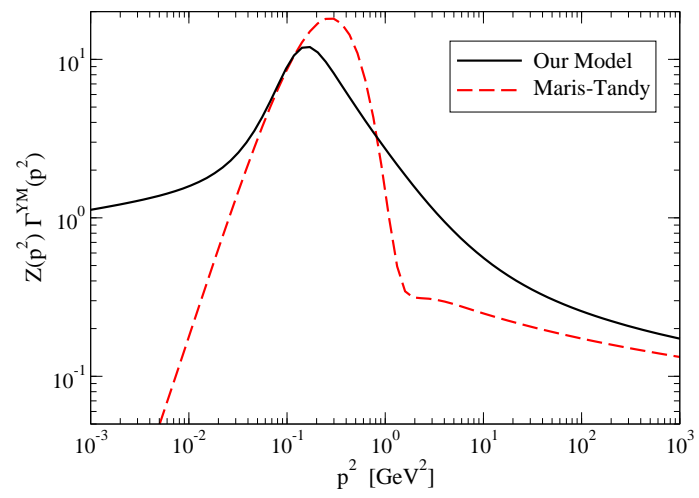

FIG. 4: A log-log plot of the effective quark-gluon interaction $Z \Gamma^{Y M}$ for the two models we employ. Differences in the UV behaviour are attributed to the different scale $\Lambda_{\mathrm{QCD}}^{2}$ in use. Note both exhibit enhancement in the intermediate momentum region.

change of slope in the infrared region of our interaction is related to the topological mass component of the $\eta^{\prime}$ in the chiral limit 33].

As for the pion part of our interaction, we approximate the full pion Bethe-Salpeter wave function (10) in the quark-SDE (5) and the kernel of the BSE (8) by the leading amplitude in the chiral limit given by [2]

$$
\Gamma_{\pi}^{j}(p ; P)=\tau^{j} \gamma_{5} \frac{B\left(p^{2}\right)}{f_{\pi}} .
$$

Here $B\left(p^{2}\right)$ is the scalar dressing function of the quark propagator in the chiral limit. This approximation omits the back-coupling effects of the three sub-leading amplitudes. From the calculation of ref. [15], where full back-coupling has been evaluated in a real value approximation, we expect this omission to lead to an error of only a few percent as concerns meson masses and of about $10-20 \%$ as concerns decay constants. Considering the exploratory character of our work this seems certainly tolerable. The replacement of the leading physical pion amplitude $F_{1}(p ; P)$ at $m_{\pi}=138 \mathrm{MeV}$ by the (normalised) chiral limit value $F_{1}(p ; P) \approx \frac{B\left(p^{2}\right)}{f_{\pi}}$ is also correct on the few percent level as demonstrated in the appendix. Note that we use this approximation only for the internal pion which mediates the interaction. The great advantage of the approximation (14) compared to the full back-coupling performed in Ref. [15] is that we can then fully take into account the quark propagator in the complex plane as necessary input into the BetheSalpeter equation.

\section{Decay constants and normalisation}

The Bethe-Salpeter amplitudes for the pseudoscalar mesons can be used to obtain the corresponding weak decay constants $f_{\pi}$, which will prove useful in fitting parameters to observables. In order to calculate these one 
must firstly normalise the amplitudes. The normalisation condition is derived by demanding that the residue of the pole in the four-point quark-antiquark Green's function (from which the BSE is derived) be unity [36, 37]. Using conventions such that $f_{\pi}=93 \mathrm{MeV}$, and for momenta partitioned equally between the two constituents, it reads

$$
\begin{aligned}
\delta^{i j}= & 2 \frac{\partial}{\partial P^{2}} \operatorname{tr} \int \frac{d^{4} k}{(2 \pi)^{4}} \\
& {\left[3\left(\bar{\Gamma}_{\pi}^{i}(k,-Q) S(k+P / 2) \Gamma_{\pi}^{j}(k, Q) S(k-P / 2)\right)\right.} \\
+ & \left.=\frac{d^{4} q}{(2 \pi)^{4}}\left[\bar{\chi}_{\pi}^{i}\right]_{s r}(q,-Q) K_{t u ; r s}^{\text {pion }}(q, k ; P)\left[\chi_{\pi}^{j}\right]_{u t}(k, Q)\right]
\end{aligned}
$$

where $Q^{2}=-M^{2}$ is fixed to the on-shell meson mass, the trace is over Dirac matrices and the Bethe-Salpeter wave-function $\chi$ is defined by

$$
\chi_{\pi}^{j}(k ; P)=S(k+P / 2) \Gamma_{\pi}^{j}(k, P) S(k-P / 2) .
$$

The conjugate vertex function $\bar{\Gamma}$ is given by

$$
\bar{\Gamma}(p,-P)=C \Gamma^{T}(-p,-P) C^{-1},
$$

with the charge conjugation matrix $C=-\gamma_{2} \gamma_{4}$. The leptonic decay constant characterising the pion coupling to the point axial field is subsequently given by 37]

$$
f_{\pi}=Z_{2} \frac{3}{M^{2}} \operatorname{tr} \int \frac{d^{4} k}{(2 \pi)^{4}} \Gamma_{\pi}(k,-P) S\left(k_{+}\right) \gamma_{5} \not P S\left(k_{-}\right),
$$

where again the trace is over Dirac matrices, and $k_{+}=$ $k+P / 2, k_{-}=k-P / 2$. There exist analogous expressions for the vector mesons [4], where an additional factor of $1 / 3$ must be included due to summation over the polarisation tensor.

One may write a similar equation to (18), which corresponds to the residue of the pseudoscalar vertex:

$$
r_{\pi}=Z_{2} Z_{m} 3 \operatorname{tr} \int \frac{d^{4} k}{(2 \pi)^{4}} \Gamma_{\pi}(k,-P) S\left(k_{+}\right) \gamma_{5} S\left(k_{-}\right) .
$$

The axWTI imposes a relationship between these two residues, known as the generalised Gell-Mann-OakesRenner relation [3], which must hold at and beyond the chiral limit:

$$
f_{\pi} m_{\pi}^{2}=r_{\pi}\left(m_{u}\left(\mu^{2}\right)+m_{d}\left(\mu^{2}\right)\right)
$$

where $m_{u}, m_{d}$ are the masses of the up and down quarks at the renormalisation point $\mu^{2}$, and in this work considered to be degenerate. Confirming this relation serves as a check of both our numerics and indicates how well our kernel satisfies the axWTI. We find excellent agreement with errors smaller than $1 \%$ for pion masses up to $m_{\pi} \simeq 600 \mathrm{MeV}$.

\section{NUMERICAL METHODS AND RESULTS}

\section{A. Solving the BSE}

We solve the BSE for the meson amplitude $\Gamma^{(\mu)}(p ; P)$ via matrix methods using the following procedure. First, we project our BSE onto the scalar amplitudes of our meson decomposition. This gives rise to either four or eight coupled integral equations for the $F_{i}(p ; P)$. To make manifest the angular dependence of the amplitude functions, we treat the total momentum $P^{2}$ as a parameter and expand the function as a series of Chebyshev polynomials in the angle $\widehat{p \cdot P}=p \cdot P /|p P|$

$$
F_{i}(p ; P)=\sum_{k}(i)^{k} F_{i}^{k}\left(p^{2} ; P^{2}\right) T_{k}(\widehat{p \cdot P}) .
$$

The functions $F_{i}^{k}\left(p^{2} ; P^{2}\right)$ are projected out through use of the orthonormal properties of the Chebyshev polynomials. With the angular dependence made explicit, we can evaluate numerically the two non-trivial angles appearing in the integration measure. We cast the remaining radial integral in the form of a matrix equation by matching the external momenta to the radial loop momenta, $p_{j}^{2}=k_{j}^{2}$ at the abscissae of our integration nodes. Thus the amplitude of our BS equation is projected onto the decomposition $F_{i}^{k}\left(p_{j}^{2} ; P^{2}\right)$. Schematically we are solving

$$
\Gamma=\lambda \mathbf{K} \cdot \Gamma
$$

for the column vector $\Gamma$ as a parametric equation in $P^{2}=-M^{2}$ with $M$ the mass of the meson. A boundstate corresponds to solutions with $\lambda=1$, which as an eigenvalue problem is equivalent to satisfying the condition $\operatorname{det}(\mathbb{1}-\mathbf{K})=0$. Because of the tractable nature of our unquenching prescription, the computational effort required to determine the Bethe-Salpeter amplitude increases from a few seconds to a few minutes. However, we see in the next section that substantial effort is required to calculate the normalisation condition with any kernel involving momentum exchange of the meson.

\section{B. Calculating the normalisation}

The first term of (15), independent of the kernel, is easily evaluated since the quark propagator derivatives are directly calculable in our approach; either by applying the derivative to the quark-SDE, and solving the resulting integral equation, or by finite difference methods. The second term is substantially more complicated for two reasons. First, we must determine the derivative of our exchange kernel with respect to the total momentum dependence - a tractable but algebraically complicated task. The second problem is the double integration over two four-momenta, $k$ and $q$. Decomposing the measure into hyperspherical coordinates, where none of the angles 
may be evaluated trivially, results in the need to evaluate an eight dimensional integral numerically. Resigning ourselves to this computationally intensive task, we evaluate the integral of (15) for $P^{2}=Q^{2} \pm 2^{n} \Delta$, and employ the appropriate Richardson improved centered difference formula. Typically, we use a four-point rule for which $n=0,1$ though a two-point rule is sufficient, provided we choose $\Delta$ optimally. Unfortunately each double integral must still be evaluated, which we tackled using both Monte-Carlo and Gaussian quadrature techniques. For the latter, a small-scale computing cluster is mandatory for even moderate grid sizes. Monte-Carlo performs only marginally better in terms of necessary computing time, since the statistical errors must be minimised such that the numerical derivative is meaningful. With due care, however, both methods obviously yield the same result.

In the next section, we give results for the leptonic decay constants for the pion and rho with and without consideration of the normalisation double-integral. For a physical pion, the difference is only about $3 \%$ and smaller still for the rho. However, as we reduce the pion mass and approach the chiral limit the necessity of including the full normalisation condition becomes readily apparent.

\section{Results}

The parameters of the two models we employed - that of Maris-Tandy and our model - were tuned such that pion observables were well reproduced with the inclusion of the hadronic exchange kernel. For our interaction, which is capable of generating a topological charge, the eta/eta' splitting provides an additional constraint.

For the Maris-Tandy model, we find a unique choice of $\omega$ and $D$ for a given quark mass by fitting to $m_{\pi}$ and $f_{\pi}$. We attempted to maximise the rho meson mass, though we did not exhaust the available parameter space due to the complexity in evaluating the full normalisation condition. Consequently we employ $\omega=0.37 \mathrm{GeV}$ and $D=1.45 \mathrm{GeV}^{2}$, with a quark mass of $3.7 \mathrm{MeV}$ at $\mu=19 \mathrm{GeV}$.

The parameters of our model interaction were fit in a similar fashion, with the additional step of calculating the topological charge for each parameter set. As a result, we obtained good agreement with experiment with the choice $d_{1}=1.45 \mathrm{GeV}^{2}, d_{2}=0.1 \mathrm{GeV}^{2}$ and $d_{3}=3.95 \mathrm{GeV}^{2}$. Here we choose a quark mass $m(\mu)=2.6 \mathrm{MeV}$ defined at the renormalisation point $\mu^{2}=170 \mathrm{GeV}^{2}$.

In Table III we present a summary of meson observables calculated for these two interactions, with and without the inclusion of the pion exchange kernel. One may then compare the effects of unquenching for two models with dissimilar behaviour in the infrared and intermediate momentum regions (see Fig. 4). Though the details of the deep infrared are generally unimportant for the pattern of dynamical symmetry breaking in light mesons, we expect a complicated interplay from the dynamics

\begin{tabular}{c|cc|cc|c} 
& \multicolumn{2}{|c|}{ Maris-Tandy } & \multicolumn{2}{|c|}{ Our Model } & Experiment \\
& w/o pi & incl. pi & w/o pi & incl. pi & (PDG [38]) \\
\hline \hline$M_{\pi}$ & 140 & $138^{\dagger}$ & 125 & $138^{\dagger}$ & 138 \\
$f_{\pi}$ & 104 & $93.2^{\dagger}$ & 102 & $93.8^{\dagger}$ & 92.4 \\
& & $(90.2)$ & & $(90.6)$ & \\
\hline$M_{\sigma}$ & 746 & 598 & 638 & 485 & $400-1200$ \\
$M_{\rho}$ & 821 & 720 & 795 & 703 & 776 \\
$f_{\rho}$ & 160 & 167 & 159 & 162 & 156 \\
& & $(167)$ & & $(165)$ & \\
$M_{a_{1}}$ & 979 & 913 & 941 & 873 & 1230 \\
$M_{b_{1}}$ & 820 & 750 & 879 & 806 & 1230 \\
\hline$M_{\eta}$ & & & 493 & 497 & 548 \\
$M_{\eta^{\prime}}$ & & & 949 & 963 & 948 \\
\hline
\end{tabular}

TABLE III: BSE results for the pseudoscalar, scalar, vector and axial-vector mesons for the Maris-Tandy and softdivergence models employed with ('incl.') and without ('w/o') pion backreaction. The parameters marked by $\dagger$ are fitted to reproduce the pion mass and decay constant when the pionexchange kernel is switched on. Consequently the model parameters, given in the text, are modified compared to the original Maris-Tandy model [4]. Results for the pure rainbowladder kernel without pion back-reaction are for the same parameter set. Decay constants given in parenthesis are those that would result without calculating the full normalisation condition of the appropriate meson.

present in any beyond-the-rainbow truncation.

This difference in the two interactions is seen most prominently for the pion. Though the effects here are small, as to be expected we see immediately a difference for the two models. Whilst for Maris-Tandy, the inclusion of pion-exchange is attractive giving rise to a $2 \mathrm{MeV}$ shift, our model exhibits repulsion of the order of $13 \mathrm{MeV}$. The leptonic decay constants see a decrease of $10 \%$ for both models. The effect of including hadronic contributions is not then a priori known, at least for those mesons most sensitive to dynamical chiral symmetry breaking.

For the remaining light mesons, the inclusion of the pion back-reaction generally leads to a lighter spectrum. In the case of the scalar meson, we see a reduction of $150 \mathrm{MeV}$ for both interactions, though the relative drop is significantly different at 20 and $40 \%$ for Maris-Tandy and our model respectively.

The vector meson - here identified with the $\rho$ - receives a negative shift of $100 \mathrm{MeV}$, or $12 \%$ for both interactions. In the case of Maris-Tandy, the resultant rho mass of $\sim 720 \mathrm{MeV}$ is similar to that obtained in other studies with this interaction [4]. Here, unquenching has the effect of increasing the decay constant by $5 \%$. Our interaction yields a lighter mass of around $700 \mathrm{MeV}$, with unquenching increasing the decay constant by just $2 \%$. Since our truncation scheme does not provide a mechanism for the $\rho$ to decay into pions, we do not observe the tell-tale non-analytic behaviour of the rho mass as a function of the pion mass, due to the opening of a decay channel, as shown in Ref. [39]. 
Next, we examine the axial-vectors $a_{1}$ and $b_{1}$, which differ by their charge conjugate properties. As already seen, the net effect of including the pion back-reaction is a reduction in the meson mass. For the axial-vectors, we see a $70 \mathrm{MeV}$ reduction in the bound-state mass for both charge eigenstates and in both models of the interaction. It is evident, at least with our tractable unquenching truncation scheme, that unquenching effects do not resolve the difficulties we have in obtaining reasonable masses for these mesons. However, we note that the anomalous mass-splitting in calculations of the $a_{1}$ and $b_{1}$ in our model is half that of Maris-Tandy. In the course of our investigations of possible parameter sets, we could easily obtain near degenerate axial-vector masses within our interaction.

Finally, we take a look at the masses of the $\eta$ and $\eta^{\prime}$. For our model, which exhibits a soft-divergence in the gluon momentum, we calculate a non-zero topological mass following Ref. [33]. We see that the inclusion of the pion back-reaction on the quark propagator has a minimal effect on the phenomenologically determined mass-splittings.

\section{CONCLUSIONS AND OUTLOOK}

Using an approximation scheme for including pion loop effects in the quark-gluon interaction, we investigated effects of the pion cloud in the Bethe-Salpeter and Schwinger-Dyson equations. By considering only the leading amplitude of the exchange pion, moreover taken from its chiral limit solution, we were able to include the analytic behaviour beyond the real-value approximation employed in the previous study [15]. The resulting interaction respects the axial-vector Ward-Takahashi identity.

As a consequence of this technical improvement, we were forced to tackle the added complication of calculating the full normalisation condition of the Bethe-Salpeter equation where the momentum exchange of the kernel need be considered. We found that this indeed gives a sizable contribution of the order of a few percent that must be properly taken into account in order to extract the correct leptonic decay constants.

As a result, we found considerable pion cloud effects in the spectrum of light mesons. In particular we found a shift of about $100 \mathrm{MeV}$ for the mass of the rho-meson in agreement with expectations from chiral extrapolations of quenched lattice data [40].

Though this truncation scheme represents a tractable model for including hadronic unquenching effects, it is clear that spin dependent contributions from the YangMills part of the quark-gluon vertex will have a strong impact upon the spectrum of light mesons. Such corrections will form a part of future investigations.

\section{Acknowledgements}

We are grateful to Michael Buballa and Dominik Nickel for fruitful discussions on the pion back-reaction. We thank Peter Tandy for a critical reading of the manuscript. This work has been supported by the Helmholtz-University Young Investigator Grant No. VHNG-332.

\section{APPENDIX A: PION AMPLITUDE AT AND AWAY FROM THE CHIRAL LIMIT}

One of the key approximations here is that the leading Bethe-Salpeter amplitude for the pion may be well approximated by the chiral limit result $B / f_{\pi}$. It is important for the amplitude to feature the correct asymptotic behaviour at large momenta, and so for a massive pion in the exchange kernel we employ the scalar self-energy $B$ as calculated in the chiral limit (for complex momenta). The differences between this and the full amplitude are

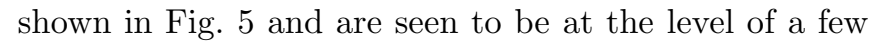
percent.

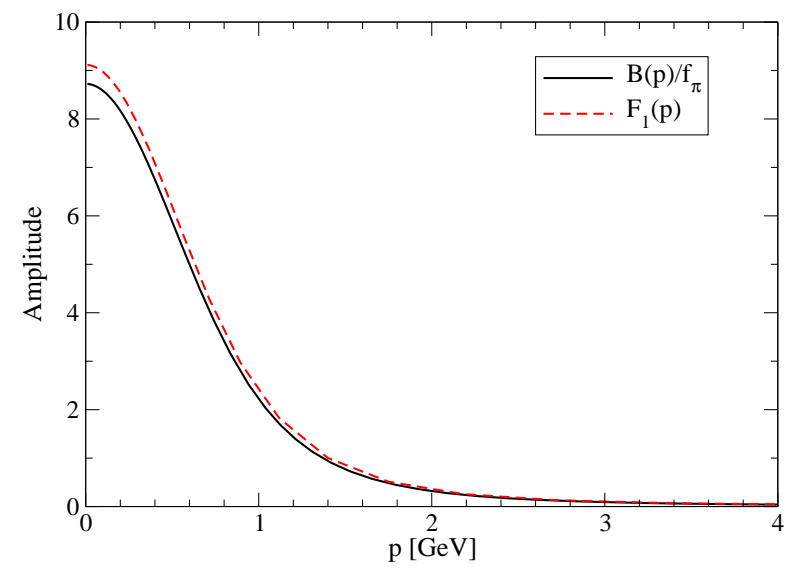

FIG. 5: The leading physical pion amplitude $F_{1}(p, P)$ at $m_{\pi}=138 \mathrm{MeV}$, compared to the normalised chiral limit value $F_{1}(p ; P) \approx \frac{B\left(p^{2}\right)}{f_{\pi}}$. The angular dependence of this amplitude is negligible, and chosen here such that $(p \cdot P)=0$.
[1] A. W. Thomas and J. Lab, Prog. Part. Nucl. Phys. 61 (2008) 219 arXiv:0805.4437 [hep-ph]].

[2] P. Maris, C. D. Roberts and P. C. Tandy, Phys. Lett. B 420 (1998) 267 arXiv:nucl-th/9707003.

[3] P. Maris and C. D. Roberts, Phys. Rev. C 56 (1997) 3369
arXiv:nucl-th/9708029

[4] P. Maris and P. C. Tandy, Phys. Rev. C 60 (1999) 055214 arXiv:nucl-th/9905056.

[5] P. Maris and P. C. Tandy, Phys. Rev. C 62, 055204 (2000) arXiv:nucl-th/0005015. 
[6] M. S. Bhagwat and P. Maris, Phys. Rev. C 77 (2008) 025203 arXiv:nucl-th/0612069.

[7] J. Volmer et al. TThe Jefferson Lab F(pi) Collaboration], Phys. Rev. Lett. 86, 1713 (2001) arXiv:nucl-ex/0010009.

[8] R. Alkofer, C. S. Fischer and F. J. Llanes-Estrada, Mod. Phys. Lett. A 23, 1105 (2008) arXiv:hep-ph/0607293.; R. Alkofer, C. S. Fischer, F. J. Llanes-Estrada and K. Schwenzer, Annals of Physics in print; arXiv:0804.3042 [hep-ph].

[9] R. Alkofer, P. Watson and H. Weigel, Phys. Rev. D 65 (2002) 094026 arXiv:hep-ph/0202053.

[10] C. Amsler and N. A. Tornqvist, Phys. Rept. 389 (2004) 61.

[11] M. R. Pennington, Int. J. Mod. Phys. A 21 (2006) 747 arXiv:hep-ph/0509265.

[12] M. Wagner and S. Leupold, Phys. Rev. D 78 (2008) 053001 arXiv:0801.0814 [hep-ph]].

[13] J. A. Oller and E. Oset, Nucl. Phys. A 620 $\begin{array}{llllll}(1997) & 438 & \text { [Erratum-ibid. } & \text { A } & \mathbf{6 5 2} & \text { (1999) }\end{array}$ arXiv:hep-ph/9702314.

[14] J. A. Oller and E. Oset, Phys. Rev. D 60 (1999) 074023 arXiv:hep-ph/9809337.

[15] C. S. Fischer, D. Nickel and J. Wambach, Phys. Rev. D 76 (2007) 094009 [arXiv:0705.4407 [hep-ph]].

[16] C. S. Fischer, D. Nickel and R. Williams, arXiv:0807.3486 [hep-ph].

[17] P. Maris and C. D. Roberts, Int. J. Mod. Phys. E 12 (2003) 297 arXiv:nucl-th/0301049.

[18] C. S. Fischer, J. Phys. G 32, R253 (2006) arXiv:hep-ph/0605173.

[19] C. S. Fischer, P. Watson and W. Cassing, Phys. Rev. D 72, 094025 (2005) arXiv:hep-ph/0509213.

[20] P. Watson, W. Cassing and P. C. Tandy, Few Body Syst. 35 (2004) 129 arXiv:hep-ph/0406340.

[21] M. S. Bhagwat, A. Holl, A. Krassnigg, C. D. Roberts and P. C. Tandy, Phys. Rev. C 70, 035205 (2004) arXiv:nucl-th/0403012.

[22] P. Maris and P. C. Tandy, Nucl. Phys. Proc. Suppl. 161, 136 (2006) arXiv:nucl-th/0511017.

[23] A. Bender, C. D. Roberts and L. Von Smekal, Phys. Lett.
B 380, 7 (1996) arXiv:nucl-th/9602012.

[24] H. H. Matevosyan, A. W. Thomas and P. C. Tandy, Phys. Rev. C 75 (2007) 045201 arXiv:nucl-th/0605057.

[25] P. Watson and W. Cassing, Few Body Syst. 35 (2004) 99 arXiv:hep-ph/0405287.

[26] C. S. Fischer and R. Alkofer, Phys. Rev. D 67 (2003) 094020 arXiv:hep-ph/0301094.

[27] M. S. Bhagwat, M. A. Pichowsky, C. D. Roberts and P. C. Tandy, Phys. Rev. C 68 (2003) 015203 arXiv:nucl-th/0304003.

[28] C. S. Fischer and M. R. Pennington, Phys. Rev. D 73 (2006) 034029 arXiv:hep-ph/0512233 ; Eur. Phys. J. A 31 (2007) 746 arXiv:hep-ph/0701123.

[29] J. Skullerud and A. Kizilersu, JHEP 0209 (2002) 013 arXiv:hep-ph/0205318.

[30] J. I. Skullerud, P. O. Bowman, A. Kizilersu, D. B. Leinweber and A. G. Williams, JHEP 0304 (2003) 047 arXiv:hep-ph/0303176; A. Kizilersu, D. B. Leinweber, J. I. Skullerud and A. G. Williams, Eur. Phys. J. C 50 (2007) 871 arXiv:hep-lat/0610078.

[31] C. H. Llewellyn-Smith, Annals Phys. 53 (1969) 521.

[32] R. Alkofer, W. Detmold, C. S. Fischer and P. Maris, Phys. Rev. D 70 (2004) 014014 arXiv:hep-ph/0309077.

[33] R. Alkofer, C. S. Fischer and R. Williams, arXiv:0804.3478 [hep-ph].

[34] D. Zwanziger, Phys. Rev. D 65, 094039 (2002) arXiv:hep-th/0109224.

[35] C. Lerche and L. von Smekal, Phys. Rev. D 65, 125006 (2002) arXiv:hep-ph/0202194.

[36] N. Nakanishi, Prog. Theor. Phys. Suppl. 43 (1969) 1.

[37] P. C. Tandy, Prog. Part. Nucl. Phys. 39 (1997) 117 arXiv:nucl-th/9705018.

[38] W. M. Yao et al. [Particle Data Group], J. Phys. G 33 (2006) 1.

[39] C. R. Allton, W. Armour, D. B. Leinweber, A. W. Thomas and R. D. Young, Phys. Lett. B 628 (2005) 125 arXiv:hep-lat/0504022.

[40] D. Leinweber et al, Talk given at the conference 'Tropical QCD' in Port Douglas, Australia, 27.7.-1.8.2008. 